\title{
DETECCIÓN DE GLAUCOMA MEDIANTE TELEMEDICINA CON SisGlaTel: SATISFACCIÓN Y ACEPTACIÓN ENTRE LOS PARTICIPANTES Y PROBLEMAS DETECTADOS
}

\section{DETECTION OF GLAUCOMA USING SisGlaTel: ACCEPTABILITY AND SATISFACTION AMONG PARTICIPANTS, AND PROBLEMS DETECTED}

\author{
BLÁZQUEZ F ${ }^{1}$, SEBASTIÁN MA², ANTÓN A ${ }^{3}$
}

\section{RESUMEN}

Propósito: Evaluar las dificultades y el grado de aceptación y satisfacción entre la población participante durante una campaña de detección de glaucoma por telemedicina. Este trabajo es parte de un estudio global de desarrollo, aplicación y evaluación del SisGlaTel financiado por el Fondo de Investigaciones Sanitarias (FIS) y Pfizer Ophthalmics.

Método: Se desarrolló un sistema de telemedicina sobre un programa específico en JAVA ${ }^{\mathrm{TM}}$. Se instaló la estación remota en el Centro de Atención Primaria. Los sujetos participantes firmaron un consentimiento informado, y fueron sometidos a varias pruebas diagnósticas (perimetría de duplicación de frecuencia-FDT, oftalmoscopia con láser confocalHRT y tonometría). Se analizaron los resultados de las pruebas y los problemas observados en la aplicación del sistema. Se realizó una encuesta a 99 personas que acababan de participar voluntariamente en el programa de detección de glaucoma entre población de riesgo. La encuesta incluyó 7 pregun-
Purpose: To evaluate the problems, and the degree of acceptability and satisfaction among participants in a telemedicine screening program. This study forms part of a global project for the design, implementation and evaluation of SisGlaTel funded by the Fondo de Investigaciones Sanitarias (Spain) and Pfizer Ophthalmics.

Method: A telemedicine system was built based on a specific program written in JAVA ${ }^{\mathrm{TM}}$. The remote station was located at a Primary Care Center. Participants signed an informed consent, and completed a questionnaire and undertook different tests (frequency doubling perimetry-FDT, confocal scanning laser tomografy-HRT and tonometry). Test results and problems detected were analysed. Ninety-nine subjects completed a satisfaction questionnaire after voluntarily participating in the screening program among an at-risk population. The questionnaire included 7 questions, four of which required participants to categorise their responses, ranging from very bad (1) to excellent (5), to questions

\footnotetext{
Recibido: 31/5/06. Aceptado: 25/8/08.

IOBA. Universidad de Valladolid. España.

1 Licenciado en Medicina.

2 Enfermera.

3 Doctor en Medicina.

Financiado por: Fondo de Investigaciones Sanitarias y Pfizer Ophthalmics.

Correspondencia:

A. Antón

Av. Bogatell, 1, 3-2

08005 Barcelona

España

E-mail: anton@icrcat.com
} 
tas. Cuatro de ellas requerían evaluar entre muy malo: 1 y excelente: 5 distintos aspectos de la campaña.

Resultados: Se evaluaron 641 sujetos con riesgo de sufrir glaucoma. El 22,3\% (143) realizaron un FDT patológico y un 4,2\% (27) presentaron un HRT patológico. El 100\% de los encuestados afirmó su deseo de volver a participar en una campaña similar. La valoración media (DS) fue de 4,5 $(0,6), 4,5$ $(0,6), 4,8(0,5)$, y 4,3 $(0,7)$ para la opinión general, la utilidad, la atención recibida, y las instalaciones, respectivamente.

Conclusión: El SisGlaTel permite integrar la información de distintas pruebas diagnósticas para detección de glaucoma por telemedicina. El grado de satisfacción entre los participantes fue bueno.

Palabras clave: Glaucoma, telemedicina, rastreo, perimetría de duplicación de frecuencia, oftalmoscopía con láser confocal. about the screening program, its usefulness, the care received and the installations.

Results: Six hundred and forty-one at-risk subjects were examined. After 2 exams per eye, 22.3\% (143) presented abnormal FDT and 4.2\% (27) had pathologic HRT. One hundred percent of respondents affirmed they would participate again in a similar program. The mean qualification (SD) was 4.5 (0.6), $4.5(0.6), 4.8(0.5)$, and 4.3 (0.7) for global opinion, usefulness, care and equipment.

Conclusion: SisGlaTel allows integration of several diagnostic tests useful for glaucoma tele-screening. The global degree of satisfaction among participants was good (Arch Soc Esp Oftalmol 2008; 83: 533-538).

Key words: Glaucoma, telemedicine, screening, frequency doubling technology, scanning laser opthalmoscopy.

\section{INTRODUCCIÓN}

El glaucoma primario de ángulo abierto es una neuropatía óptica frecuente, $2,1 \%$ de la población mayor de 40 años en nuestro entorno (1), y se caracteriza por una pérdida progresiva de fibras nerviosas y deterioro de la función visual que puede llegar a provocar una ceguera irreversible. Se trata de una enfermedad asintomática para el paciente hasta que llega a estadios avanzados, y los medios terapéuticos disponibles actualmente (fármacos, láser, y cirugía) sólo permiten retrasar o detener su evolución. Por todas estas razones, el glaucoma es una de las primeras causas de ceguera en la sociedad industrializada (6 al 21\% de las causas de ceguera), más de la mitad de los casos no se encuentran diagnosticados, y su mejor tratamiento es el diagnóstico precoz. La Organización Mundial de la Salud recomienda en sus programas de prevención de la ceguera la toma de medidas específicas para el diagnóstico precoz de esta enfermedad.

La telemedicina consiste en la utilización de la tecnología de las telecomunicaciones para proporcionar cuidados médicos oftalmológicos. Permite acercar la más avanzada tecnología y los conocimientos de las consultas más especializadas a la población. La telemedicina tiene un gran potencial en áreas rurales o alejadas de atención médica especializada, para evitar desplazamiento de pacientes y personal facultativo, para acercar la tecnología y el conocimiento a la población, y para la aplicación de programas de rastreo.

Una de las aplicaciones de la telemedicina más desarrolladas en oftalmología es la realización de campañas de prevención de la ceguera por retinopatía diabética. Existen estudios recientes sobre la aplicación de la telemedicina al glaucoma que han evaluado la presión ocular o han utilizado distintos sistemas de fotografía o vídeo (2-7). Se han utilizado instrumentos de imagen para la detección de glaucoma por telemedicina $(7,8)$ pero no se han encontrado en la literatura referencias acerca de la aplicación de la oftalmoscopía con láser confocal y la perimetría de duplicación de frecuencia. La sensibilidad y especificidad obtenidas tras la evaluación subjetiva, por uno o varios oftalmólogos, de fotografías de la papila se cifra en $70 \%$ y $97 \%$ respectivamente (6).

La incorporación de instrumentos diagnósticos capaces de obtener imágenes tridimensionales de la papila y una evaluación funcional rápida y sensible podrían aumentar las posibilidades diagnósticas de los sistemas de telemedicina. Además estos instrumentos son relativamente fáciles de manejar y no requieren la participación de un oftalmólogo. Una enfermera, optómetra o técnico entrenado podrían manejarlos y sus resultados pueden ser enviados por internet en forma de fichero. El programa TOSCA 
2002 para la detección de glaucoma, realizado en varios países europeos, fue el primero en incorporar la oftalmoscopía con láser confocal a programas de telemedicina. No se ha encontrado ninguna publicación sobre este programa europeo.

La integración de una breve anamnesis, la medida de la presión ocular, una prueba funcional y una prueba estructural breves, pero precisas, podría ser particularmente eficaz en un sistema de detección de glaucoma aplicable a distancia por telemedicina. Este sistema podría ayudar a detectar los muchos casos de glaucoma sin diagnosticar y a prevenir la ceguera por esta enfermedad.

El presente proyecto pretende evaluar las dificultades y el grado de aceptación y satisfacción entre la población participante durante la aplicación del sistema de telemedicina SisGlaTel en una campaña de detección de glaucoma. Este trabajo es parte de un estudio global de desarrollo, aplicación y evaluación del SisGlaTel financiado por el FIS y Pfizer Ophthalmics.

\section{SUJETOS, MATERIAL Y MÉTODO}

\section{SisGlaTel}

El sistema de telemedicina SisGlaTel se desarroló sobre un programa específico en JAVA ${ }^{\mathrm{TM}}$ que integra varias pruebas de diagnóstico de glaucoma. El esquema global del SisGlaTel se representa en la figura 1. El programa permite mantener la comunicación entre uno o varios centros remotos y un centro de lectura y proceso de datos donde se encuentra la base de datos (MySQL). El sistema se ha construido sobre una base multi-plataforma y con programas gratuitos. Emplea un servidor de aplicaciones en la web Apache Tomcat donde residen todos los procesos de comunicación entre el cliente y el servidor. El sistema cumple con la normativa sobre protección de datos.

Los datos de filiación y la presión se introducen manualmente y los ficheros con datos de la perimetría de duplicación de frecuencia (FDT, HumphreyZeiss) y del Heidelberg Retina Tomograph (HRT, Heidelberg Engineering) se importan automáticamente. Los datos son transmitidos al servidor vía internet una vez encriptados y fragmentados. El ordenador cliente no almacena datos. En el servidor los datos se integran, se reconstruyen los resultados del FDT y el HRT, y son acumulados en la base de datos. Allí son revisados por un oftalmólogo que

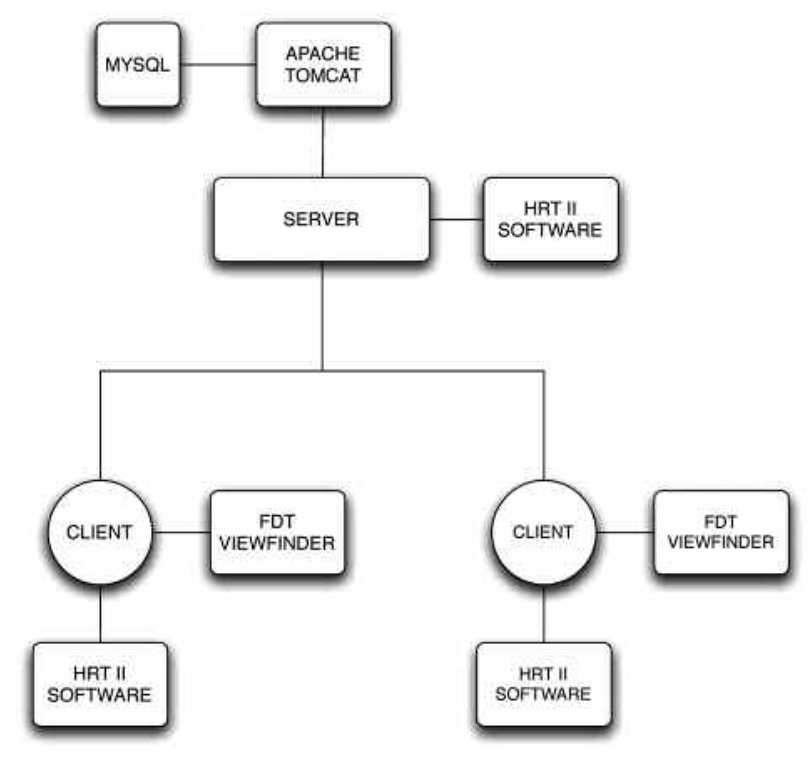

Fig. 1: Esquema general del SisGlaTel. Un programa específico permite la conexión entre el servidor y los centros remotos mediante una línea ADSL.

comprueba la calidad de los mismos y emite un informe.

En la versión inicial de este sistema, que está siendo revisada, se clasificaron como patológicos todos los HRT que resultaron fuera de los límites de la normalidad con el Análisis de Regresión de Moorfields implementado en el HRT. Así mismo, fueron considerados patológicos los FDT con 3 puntos contiguos fuera del percentil 95 de la normalidad y localizados en mismo hemicampo. De acuerdo con los resultados el oftalmólogo (AA) emitió un informe aconsejando evaluación oftalmológica si el FDT y/o el HRT eran patológicos y una evaluación periódica si la presión era superior a 21 mmHg y las pruebas se encontraban dentro de los límites de la normalidad.

\section{Aplicación en población diana}

El centro remoto se instaló en el Centro de Atención Primaria Plaza del Ejército de Valladolid y el servidor en la Clínica de la Universidad. Se colocaron paneles y trípticos informativos para solicitar la colaboración voluntaria de sujetos con riesgo de padecer glaucoma. Concretamente se incluyeron en la campaña personas mayores de 65 años y aquellos que siendo mayores de 40 años tuvieran anteceden- 
tes personales de hipertensión ocular o antecedentes familiares de glaucoma. Las personas interesadas acudieron al mostrador de información o llamaron a los números indicados en carteles y trípticos. El personal encargado de la campaña (enfermeras y optómetras) contactó con las personas interesadas y mediante una breve entrevista telefónica comprobó que cumplían los criterios de inclusión y asignó una cita. Los participantes firmaron un consentimiento informado y todos realizaron un cuestionario de salud, y fueron examinados con un refractómetro automático, un HRT, un FDT y un tonómetro de aire sin contacto (INDO). Se tomaron al menos dos imágenes con el HRT y se eligió la de mejor calidad evaluando la desviación estándar, el centrado, y el foco. Todos los pacientes realizaron primero un test C-205 supraumbral para familiarizarse con la prueba y después un test N-30 que fue considerado para analizar los resultados. Si alguno de los índices de fiabilidad resultó superior al 30\%, la prueba fue repetida.

\section{Encuesta de satisfacción}

Se realizó una encuesta a 99 personas que acababan de participar voluntariamente en el programa detección de glaucoma entre población de riesgo. La encuesta incluyó 7 preguntas (fig. 2). Cuatro de ellas requerían evaluar con una de cinco categorías, entre muy malo (1) y excelente (5), la campaña en general, su utilidad, la atención recibida y las instalaciones. En otra se preguntaba si la persona volvería a participar en otra campaña similar. Finalmente se pidieron comentarios y sugerencias para mejorar la campaña.

\section{Análisis de datos}

Se registraron los tiempos de exploración totales y parciales, así como las incidencias o problemas de encontrados. Los datos fueron analizados con estadística descriptiva.

\section{RESULTADOS}

Participaron en la campaña 641 sujetos, 400 mujeres (63\%) y 241 hombres (37\%). Su edad media (DE) fue de 65,4 $(10,5)$ años y la presión intraocular media (DE) era de $16,2(3,8) \mathrm{mmHg}$ (rango: 5 a $29 \mathrm{mmHg}$ ). Tras dos exámenes con el

1. ¿Qué opinión le merece la campaña de detección de glaucoma en la que ha participado?
Excelente $\square$
Muy buena $\square$
Buena $\square$
Regular $\square$
Mala $\square$

2. ¿Volvería a participar en una campaña similar?
Sí $\square$
NO $\square$
¿Por qué?

3. Utilidad de la campaña.
Excelente
Muy buena
Buena
Regular
Mala $\square$

4. ¿Cómo calificaría la atención recibida por el personal de la campaña?
Excelente
Muy buena
Buena $\square$
Regular $\square$
Mala

5. ¿Cómo calificaría las instalaciones de la campaña?
Excelente $\square$
Muy buena $\square$
Buena $\square$
Regular $\square$
Mala $\square$

6. ¿Qué aspectos de la campaña cree que deben ser mejorados?

7. Comentarios adicionales:

Fig. 2: Contenido de la encuesta de satisfacción dirigida a 99 participantes en la campaña. 
FDT, el 22,3\% de los sujetos (n: 143) presentaban un FDT patológico. El HRT resultó patológico en el $4,2 \%$ de los incluidos en el estudio (n: 27). No se pudieron obtener imágenes de buena calidad con el HRT en 31 sujetos (4,8\%). Veinticuatro pacientes tenían FDT y HRT patológicos de forma simultánea $(3,7 \%)$. La presión ocular se encontró por encima de $21 \mathrm{mmHg}$ en al menos un ojo en $17,1 \%$ de las personas participantes (n: 110). El tiempo medio (DE) de exploración global por paciente fue de 49 (18) minutos, y osciló entre 25 y 115 minutos.

El $100 \%$ de los encuestados afirmó su deseo de volver a participar en una campaña similar. La valoración media (DS) fue de 4,5 (0,6), 4,5 (0,6), 4,8 $(0,5)$, y 4,3 $(0,7)$ para la opinión general, la utilidad, la atención recibida, y las instalaciones, respectivamente. Entre las sugerencias aportadas se encontraban la realización de campañas de detección de forma periódica y, a su vez, más prolongadas para que un mayor número de personas pudieran acceder a ellas.

Entre los problemas detectados cabe resaltar los siguientes. En primer lugar, la necesidad de entrenar específicamente y motivar al personal de Atención Primaria para que las exploraciones sean fiables, rápidas y útiles para la detección. Los instrumentos empleados son relativamente sencillos de utilizar pero exigen cierta experiencia que requiere días, más que horas, de utilización para quien no los conoce. En segundo lugar se comprobó que es necesario contratar una línea ADSL con Internet Protocol (IP) fijo, y no variable como se contrata habitualmente, para poder aplicar los protocolos de seguridad del sistema de telemedicina. En tercer lugar, las personas sin experiencia perimétrica previa necesitan tiempo relativamente prolongado para realizar pruebas psicofísicas, lo que prolonga hasta una media de 50 minutos el tiempo global de exploración siguiendo el protocolo inicialmente diseñado. Además, múltiples detalles del funcionamiento del programa informático que permite manejar el sistema deben ser pulidos para que su uso resulte más sencillo, más flexible, asequible a personal auxiliar/administrativo y aplicable en la clínica y en grandes poblaciones.

\section{DISCUSIÓN}

La telemedicina se está utilizando con distintas finalidades para reducir costes sanitarios y mejorar la calidad de la atención. En oftalmología se está empleando sobre todo en la detección de enfermedades retinianas y su desarrollo ha sido mucho mayor en EEUU que en nuestro entorno. Aunque existen algunos estudios sobre la aplicación de telemedicina en el glaucoma, no hemos encontrado en la literatura herramientas específicas que integraran la aplicación de un método de imagen con una prueba psicofísica para el diagnóstico de glaucoma. El presente estudio es parte de un estudio global de desarrollo, aplicación y evaluación del SisGlaTel. Concretamente está dedicado a la evaluación del grado de satisfacción de los participantes, y la descripción de los problemas encontrados en la aplicación del sistema en $\mathrm{n}$ a población diana.

La mayoría de las experiencias previas sobre diagnostico de glaucoma por telemedicina se basan en la interpretación de fotografías del nervio óptico (2-6). Belait y colaboradores utilizaron el HRT para la detección de glaucoma y compararon distintos grados de compresión de las imágenes y demostraron que una compresión de 10:1 permite transmitir imágenes de suficiente calidad para su evaluación y clasificación (9). De Mul y colaboradores utilizaron la polarimetría con láser confocal (GDx) e imágenes obtenidas por optómetras para detectar glaucoma (8). La calidad de las imágenes resultó adecuada en un $89 \%$ los casos y el $27 \%$ de los casos evaluados fueron citados en la consulta especializada para evaluación adicional. La cifra es similar a la obtenida en el presente estudio en el que un $24 \%$ de los participantes presentaron resultados patológicos y se recomendó, para ellos, consulta oftalmológica. El grado de aceptación y satisfacción de las personas participantes en la campaña fue entre muy bueno y excelente.

Entre los problemas encontrados destaca el elevado tiempo global de exploración en la configuración inicial del sistema. La inclusión de una prueba funcional y una prueba estructural en el protocolo de detección ofrece mejores resultados de precisión diagnóstica. Sin embargo, cincuenta minutos pueden ser adecuados para una exploración completa en una consulta especializada en glaucoma pero no para realizar campañas de detección en grupos de riesgo numerosos. Se eligió el programa N30 del FDT porque estudios previos han demostrado su mayor sensibilidad en fases tempranas del glaucoma (10), en comparación con programas más cortos que utilizan una estrategia supraumbral (C-20-5). Sin embargo, el tiempo de exploración y el proceso de aprendizaje quizá resulten algo largos para ser 
aplicados en amplias poblaciones sin experiencia perimétrica previa. Estos hallazgos confirman la necesidad de una evaluación coste-efectividad del SisGlaTel, una vez incorporadas las mejoras en curso, para determinar los aspectos económico-sanitarios de su aplicación en población de riesgo.

En resumen, el SisGlaTel permite la integración de varias pruebas de diagnóstico de glaucoma y la conexión de uno o varios centros remotos con un servidor. Los problemas observados en el presente estudio están siendo utilizados para mejorar este sistema de detección.

\section{BIBLIOGRAFÍA}

1. Antón A, Andrada MT, Mujica V, Calle MA, Portela J, Mayo A. Prevalence of primary open angle glaucoma in a Spanish population: the Segovia study. J Glaucoma 2004; 13: 371-376.

2. Jamara RJ, Denial A, Valentini D, Thorn F. Clinical quality assessment using computer monitor photo images of optic nerve head cupping. Optom Vis Sci 2000; 77: 433 436.

3. Li HK, Tang RA, Oschner K, Koplos C, Grady J, Crump
WJ. Telemedicine screening of glaucoma. Telemed $J$ 1999; 5: 283-290.

4. Beauregard D, Lewis J, Piccolo M, Bedell H. Diagnosis of glaucoma using telemedicine-the effect of compression on the evaluation of optic nerve head cup-disc ratio. $J$ Telemed Telecare 2000; 6: S123-S125.

5. Tuulonen A, Ohinmaa T, Alanko HI, Hyytinen P, Juutinen A, Toppinen E. The application of teleophthalmology in examining patients with glaucoma: a pilot study. J Glaucoma 1999; 8: 367-373.

6. Yogesan K, Constable IJ, Barry CJ, Eikelboom RH, Morgan W, Tay-Kearney $M L$, et al. Evaluation of a portable fundus camera for use in the teleophthalmologic diagnosis of glaucoma. J Glaucoma 1999; 8: 297-304.

7. Lamminen H. Picture archiving and fundus imaging in a glaucoma clinic. J Telemed Telecare 2003; 9: 114-116.

8. de Mul M, de Bont AA, Reus NJ, Lemij HG, Berg M. Improving the quality of eye care with tele-ophthalmology: shared-care glaucoma screening. J Telemed Telecare 2004; 10: 331-336.

9. Belair ML, Fansi AK, Descovich D, Leblanc AR, Harasymowycz $P$. The effect of compression on clinical diagnosis of glaucoma based on non-analyzed confocal scanning laser ophthalmoscopy images. Ophthalmic Surg Lasers Imaging 2005; 36: 323-326.

10. Andrada MT, Bernaldo de Quirós P, Villegas RS, Antón A. Precisión diagnóstica de la perimetría de duplicación de frecuencia. Arch Soc Esp Oftalmol 2001; 76: 711-718. 